\title{
Mitochondrial complex I dysfunction induced by cocaine and cocaine plus morphine in brain and liver mitochondria
}

\author{
Teresa Cunha-Oliveira ${ }^{a}{ }^{*}$, Lisbeth Silva ${ }^{a}$, Ana Maria Silva ${ }^{a}$, António J. Moreno ${ }^{c}$, \\ Catarina R. Oliveira ${ }^{\mathrm{a}, \mathrm{b}}$, Maria S. Santos ${ }^{\mathrm{a}, \mathrm{c}}$ \\ ${ }^{a}$ CNC, Center for Neuroscience and Cell Biology, University of Coimbra, Largo Marquês de Pombal, 3004-517 Coimbra, Portugal \\ ${ }^{\mathrm{b}}$ Faculty of Medicine, University of Coimbra, 3004-504 Coimbra, Portugal \\ ${ }^{\mathrm{c}}$ Life Sciences Department, Faculty of Sciences and Technology, University of Coimbra, Apartado 3046, 3001-401 Coimbra, Portugal
}

\section{H I G H L I G H T S}

- Brain and liver mitochondrial respiration is differentially affected by the drugs.

- Cocaine-induced inhibition of complex I is more evident in brain mitochondria.

- Dependence on complex I may explain differences in brain and liver mitochondria.

- The drug combination had a greater effect on brain state 3 than the drugs per se.

- In other parameters the drug combination had similar effects to cocaine per se.

\section{A R T I C L E I N F O}

\section{Article history:}

Received 7 February 2013

Received in revised form 18 March 2013

Accepted 21 March 2013

Available online xxx

\section{Keywords:}

Mitochondria

Cocaine

Morphine

Speedball

Complex I

\begin{abstract}
A B S T R A C T
Mitochondrial function and energy metabolism are affected in brains of human cocaine abusers. Cocaine is known to induce mitochondrial dysfunction in cardiac and hepatic tissues, but its effects on brain bioenergetics are less documented. Furthermore, the combination of cocaine and opioids (speedball) was also shown to induce mitochondrial dysfunction. In this work, we compared the effects of cocaine and/or morphine on the bioenergetics of isolated brain and liver mitochondria, to understand their specific effects in each tissue. Upon energization with complex I substrates, cocaine decreased state- 3 respiration in brain (but not in liver) mitochondria and decreased uncoupled respiration and mitochondrial potential in both tissues, through a direct effect on complex I. Morphine presented only slight effects on brain and liver mitochondria, and the combination cocaine+morphine had similar effects to cocaine alone, except for a greater decrease in state- 3 respiration. Brain and liver mitochondrial respirations were differentially affected, and liver mitochondria were more prone to proton leak caused by the drugs or their combination. This was possibly related with a different dependence on complex I in mitochondrial populations from these tissues. In summary, cocaine and cocaine+morphine induce mitochondrial complex I dysfunction in isolated brain and liver mitochondria, with specific effects in each tissue.
\end{abstract}

(C) 2013 Elsevier Ireland Ltd. All rights reserved.

\section{Introduction}

Cocaine abuse has been associated with mitochondrial dysfunction and impairment of energy metabolism in brain (Lehrmann et al., 2003), liver (Devi and Chan, 1997) and heart (Yuan and Acosta Jr., 2000) cells. The mitochondrial respiratory chain seems to

Abbreviations: BSA, bovine serum albumin; Coc+Mor, combination of cocaine and morphine; $\triangle \Psi$, mitochondrial transmembrane potential; FCCP, carbonyl cyanide 4-(trifluoromethoxy)phenylhydrazone; RCR, respiratory control ratio; $\mathrm{TPP}^{+}$, tetraphenylphosphonium.

* Corresponding author. Tel.: +351 239820 190; fax: +351 239822776.

E-mail addresses: mteroliv@cnc.cj.uc.pt, teresa.oliveira@gmail.com (T. Cunha-Oliveira). mediate acute cocaine toxicity, as suggested by the partial resistance to cocaine toxicity in cells lacking functional mitochondria (rho-zero), when compared with rho-plus cells (Cunha-Oliveira et al., 2006). In addition, the neurotoxicity of cocaine was previously shown to involve mitochondrial dysfunction and activation of the mitochondrial apoptotic pathway (Cunha-Oliveira et al., 2006, 2010), and exposure to cocaine was found to down-regulate mitochondrial gene expression, including several subunits of NADH dehydrogenase (complex I), in rat cingulate cortex (Dietrich et al., 2004).

Cocaine may interact directly with mitochondria and other intracellular targets (Heard et al., 2008), after entering the cell due to its positive charge at physiological pH (Cunha-Oliveira et al., 2008). Studies in hepatic mitochondria showed that in vivo cocaine administration decreased state 3 respiration, the respiratory 
control ratio (RCR) and the activity of complexes I, II/III, and IV (Devi and Chan, 1997). However, much less is known about the direct effects of cocaine on the bioenergetics of brain mitochondria.

Cocaine effects may be altered if cocaine is used together with other drugs, such as opiates, a popular drug combination known as speedball (European Monitoring Center for Drugs and Drug Addiction, 2009) that has been reported to be more rewarding in rats than cocaine or heroin alone (Ranaldi and Munn, 1998). The popularity of this drug combination may be due to the reduction of the unwanted side-effects, due to complementary mechanisms of action, and/or from enhanced effects of the combination (Leri et al., 2003). Speedball has serious health consequences. The use of cocaine in combination with heroin is generally associated with a mental illness and may aggravate underlying psychological problems, such as bipolar disorder (European Monitoring Center for Drugs and Drug Addiction, 2008). In addition, speedball abusers present more severe psychopathology in comparison with other cocaine addicts, and are more likely to fail in drug abuse treatment (Bandettini Di Poggio et al., 2006).

Interestingly, a chemical interaction between cocaine and morphine was found to occur in cocaine-heroin mixtures, involving the formation of a cocaine-morphine adduct (Garrido et al., 2007), which may play a role in the effects of cocaine-heroin combinations, especially when the co-abuse occurs simultaneously and chemical interactions may occur. Thus, besides pharmacodynamic interactions, chemical interactions between drugs could also play a role in speedball neurotoxicity (Cunha-Oliveira et al., 2010). Previously, we used primary cultures of rat cortical neurons exposed to heroin and/or cocaine, administered sequentially or simultaneously (Cunha-Oliveira et al., 2010) to investigate this possibility. The effects of cocaine seemed to predominate over heroin effects when the cells were exposed to a combination of both drugs, but the extent of mitochondrial dysfunction induced by cocaine-morphine combinations was found to differ from that induced by each drug alone, in rat cortical neurons (Cunha-Oliveira et al., 2010). Moreover, the neurotoxic pathways induced by simultaneous coadministration of cocaine and heroin differed from those evoked by a sequential administration of the same drugs, possibly due to chemical interactions between the two drugs (or their metabolites), which may interfere with cell death mechanisms and may affect the capacity of cocaine to interact with its cellular targets, such as mitochondria.

In order to understand the specific effects of cocaine, morphine and cocaine+morphine in brain and liver mitochondria, in this work, we compared their effects on the bioenergetics of mitochondria isolated from each tissue.

\section{Materials and methods}

\subsection{Chemicals}

Cocaine hydrochloride and morphine sulfate salt pentahydrate were obtained from Sigma-Aldrich Co. (St. Louis, MO, USA), upon authorization by INFARMED, Portugal (National Authority of Medicines and Health Products). All of the other chemicals were of the highest grade of purity commercially available.

\subsection{Animals}

Male Wistar-Han rats (9-12 weeks old) were maintained in our animal house, under controlled light ( $12 \mathrm{~h}$ day/night cycle), temperature and humidity, with ad libitum access to food and water. Procedures were approved by the Institutional Animal Care and Use Committee.

\subsection{Isolation of liver and brain mitochondria}

Animals were sacrificed by cervical displacement and decapitation, following procedures approved by the Institutional Animal Care and Use Committee.

Liver mitochondria were isolated by conventional methods (Moreno et al., 2007), with slight modifications. Briefly, animals were sacrificed and the liver was immediately excised, finely minced, and homogenized in ice-cold medium containing $250 \mathrm{mM}$ sucrose, $10 \mathrm{mM}$ HEPES, $0.5 \mathrm{mM}$ EGTA and $0.1 \%$ defatted bovine serum albumin (BSA) ( $\mathrm{pH} 7.4)$. The homogenate was centrifuged at $900 \times g$ (Sorvall RC-5B Refrigerated Superspeed Centrifuge) for $10 \mathrm{~min}$ at $4{ }^{\circ} \mathrm{C}$. The resulting supernatant was spun at $10,000 \times \mathrm{g}$ for $10 \mathrm{~min}\left(\right.$ at $4{ }^{\circ} \mathrm{C}$ ) to pellet mitochondria. The mitochondrial pellet was washed twice $(2 \times 10,000 \times \mathrm{g}$ for $10 \mathrm{~min})$ and suspended in the washing medium (250 mM sucrose, $10 \mathrm{mM}$ HEPES, pH 7.4; EGTA and BSA were omitted from the washing medium). Protein content was determined by the Biuret method (Gornall et al., 1949), using BSA as a standard.

Brain mitochondria were isolated by the method of Rosenthal et al. (1987), with slight modifications, using $0.02 \%$ digitonin to free mitochondria from the synaptosomal fraction. Briefly, the whole brain except the cerebellum was rapidly removed, washed, minced, and homogenized at $4{ }^{\circ} \mathrm{C}$ in $10 \mathrm{ml}$ of isolation medium $(225 \mathrm{mM}$ mannitol, 75 mM sucrose, 5 mM HEPES, 1 mM EGTA, 1 mg/ml defatted BSA, pH 7.4) containing $5 \mathrm{mg}$ of the bacterial protease type VIII. Single brain homogenates were brought to $30 \mathrm{ml}$ and then centrifuged at $740 \times g$ (Sorvall RC-5B Refrigerated Superspeed Centrifuge) for $5 \mathrm{~min}$. The pellet, including the fluffy synaptosomal layer, was resuspended in $10 \mathrm{ml}$ of the isolation medium containing $0.02 \%$ digitonin and centrifuged at $11,950 \times \mathrm{g}$ for $10 \mathrm{~min}$. The brown mitochondrial pellet without the synaptosomal layer was then resuspended in $10 \mathrm{ml}$ of medium and centrifuged again at $10,000 \mathrm{rpm}$ for $5 \mathrm{~min}$. The pellet was then resuspended in $10 \mathrm{ml}$ of resuspension medium (225 mM mannitol, $75 \mathrm{mM}$ sucrose, $5 \mathrm{mM}$ HEPES, pH 7.4) and centrifuged at $11,950 \times \mathrm{g}$ for $5 \mathrm{~min}$. Finally, the mitochondrial pellet was resuspended in $200 \mu \mathrm{l}$ of resuspension medium. Protein content was determined by the Biuret method (Gornall et al., 1949), using BSA as a standard.

\subsection{Mitochondrial respiration}

Mitochondrial oxygen consumption was monitored polarographically at $30^{\circ} \mathrm{C}$ with a Clark-type oxygen electrode (Yellow Spring Instrument, Model YSI5331) connected to a Kipp and Zonen recorder accordingly to Estabrook (1967), in a thermostatic water-jacketed chamber with magnetic stirring. The reactions were carried out at $30^{\circ} \mathrm{C}$ in $1 \mathrm{ml}$ of standard respiratory medium with $1 \mathrm{mg}$ of liver mitochondria or $0.5 \mathrm{mg}$ of brain mitochondria. For liver mitochondria the reaction medium was composed of $130 \mathrm{mM}$ sucrose, $50 \mathrm{mM} \mathrm{KCl}, 2.5 \mathrm{mM} \mathrm{MgCl}_{2}, 2.5 \mathrm{mM}$ $\mathrm{KH}_{2} \mathrm{PO}_{4}, 5 \mathrm{mM}$ HEPES and $0.01 \mathrm{mM}$ EGTA ( $\mathrm{pH} 7.4$ ). For brain mitochondria the reaction medium was composed of $100 \mathrm{mM}$ sucrose, $100 \mathrm{mM} \mathrm{KCl}, 2 \mathrm{mM} \mathrm{KH}_{2} \mathrm{PO}_{4}$, $5 \mathrm{mM}$ HEPES and $0.01 \mathrm{mM}$ EGTA ( $\mathrm{pH} 7.4$ ). Liver or brain mitochondria were incubated, for $1 \mathrm{~min}$, in $1 \mathrm{ml}$ of respiratory medium in the absence or presence of the drugs, alone or combined at $0.2-1 \mathrm{mM}$, and energized with $10 \mathrm{mM}$ glutamate $/ 5 \mathrm{mM}$ malate or $5 \mathrm{mM}$ succinate. When succinate was used as respiratory substrate, the medium was supplemented with $2 \mu \mathrm{M}$ rotenone. State 3 was initiated with ADP ( $150 \mathrm{nmol} / \mathrm{mg}$ protein for brain mitochondria and $125 \mathrm{nmol} / \mathrm{mg}$ protein for liver mitochondria), followed by addition of oligomycin $(1 \mu \mathrm{g} / \mathrm{ml})$ plus $\operatorname{ADP}(150 \mathrm{nmol} / \mathrm{mg}$ protein for brain mitochondria and $125 \mathrm{nmol} / \mathrm{mg}$ protein for liver mitochondria). Uncoupled respiration was initiated by the addition of $1 \mu \mathrm{M}$ carbonyl cyanide 4-(trifluoromethoxy)phenylhydrazone (FCCP). Respiration rates were calculated assuming an oxygen concentration of $240 \mathrm{nmol} \mathrm{O}_{2} / \mathrm{ml}$ in the experimental medium at $30^{\circ} \mathrm{C}$. The respiratory state 2 (oxygen consumption in the absence of substrate), state 3 (oxygen consumption in the presence of substrate and ADP), state 4 (oxygen consumption after ADP phosphorylation) and RCR (state 3/state 4) were calculated according to Chance and Williams (1956). ADP/O ratio was expressed by the ratio between the amount of ADP added and the oxygen consumed during state 3 respiration.

\subsubsection{Assessment of mitochondrial respiratory activity through complexes I and II}

For the assessment of direct effects of the drugs and their combination over mitochondrial complexes I and II, independently of mitochondrial physiology (Moreira et al., 2012), after isolation, mitochondrial preparations were frozen in liquid nitrogen and kept at $-80^{\circ} \mathrm{C}$ until further use. At the day of the experiments, the preparations were thawed and sonicated 4 times for $10 \mathrm{~s}$, on ice, with even intervals. Oxygen consumption was measured in the oxygen electrode in $1 \mathrm{ml}$ reaction medium, using $1 \mathrm{mg}$ and $0.8 \mathrm{mg}$ protein, respectively, of liver or brain mitochondrial fractions. To evaluate the direct effect of the drugs on complex I-driven respiration, $1 \mathrm{mM}$ NADH was added. Complex II-driven respiration was assessed independently, by adding $5 \mathrm{mM}$ succinate in the presence of $2 \mu \mathrm{M}$ rotenone. $\mathrm{KCN}$ was added at the end of the experiment, to confirm oxygen consumption through complex IV.

\subsection{Membrane potential $(\Delta \Psi)$ measurements}

The mitochondrial transmembrane potential $(\Delta \Psi)$ was monitored by evaluating transmembrane distribution of the lipophilic cation $\mathrm{TPP}^{+}$(tetraphenylphosphonium) with a $\mathrm{TPP}^{+}$-selective electrode prepared according to Kamo et al. (1979) using $\mathrm{Ag} / \mathrm{AgCl}_{2}$-saturated electrode as reference. $\mathrm{TPP}^{+}$uptake was assessed from the decreased $\mathrm{TPP}^{+}$concentration in the medium sensed by the electrode. The difference between the potential of the selective electrode and the reference electrode was measured with an electrometer and recorded continuously in a Kipp and Zonen recorder. The voltage response of the $\mathrm{TPP}^{+}$electrode to $\log \left[\mathrm{TPP}^{+}\right]$was linear with a slope of $59 \pm 1$, in a good agreement with Nernst equation.

To monitor $\Delta \Psi$ associated to mitochondrial respiration, $1 \mathrm{mg}$ (liver) or $0.5 \mathrm{mg}$ (brain) mitochondria were incubated in the standard respiratory medium 
supplemented with $3 \mu \mathrm{M} \mathrm{TPP}{ }^{+}$(and $2 \mu \mathrm{M}$ rotenone if succinate was the respiratory substrate), at $30^{\circ} \mathrm{C}$, in the absence or presence of different concentrations of the drugs alone or combined, for $1 \mathrm{~min}$, prior to the addition of $10 \mathrm{mM}$ glutamate $/ 5 \mathrm{mM}$ malate or $5 \mathrm{mM}$ succinate to start the reaction. After steady-state distribution of $\mathrm{TPP}^{+}$had been reached, ADP $(150 \mathrm{nmol} / \mathrm{mg}$ protein for brain mitochondria and $125 \mathrm{nmol} / \mathrm{mg}$ protein for liver mitochondria) was added and $\Delta \Psi$ fluctuations recorded.

\subsection{Statistical analysis}

Data are expressed as mean \pm SEM and evaluated by one-way analyses of variance (ANOVA) followed by Bonferroni multiple comparison tests. Differences were considered to be statistically significant at $p$ value lower than 0.05 . All analyses were performed using GraphPad Prism 5.0 for Windows.

\section{Results}

\subsection{Respiration of brain and liver mitochondria is differentially affected by cocaine and/or morphine}

In liver mitochondria energized with complex I or complex II substrates, cocaine $(1 \mathrm{mM})$ and its combination with morphine $(1 \mathrm{mM})$ increased state 2 and 4 respiration (Fig. 1B and F). Cocaine increased state 2 respiration by $\sim 33 \%(p<0.001)$, for complex I, and by $\sim 21 \%(p<0.001)$ for complex II, whereas the combination of cocaine and morphine (Coc+Mor) increased state 2 respiration by $\sim 24 \%$ ( $p<0.001$ ) for complexes I and II (Fig. 1B). Cocaine increased state 4 respiration by $\sim 25 \%$ ( $p<0.001$ ), for complex I, and by $\sim 36 \%$ $(p<0.001)$ for complex II, whereas Coc+Mor increased state 4 respiration by $\sim 17 \%(p<0.05)$ for complex I and $\sim 29 \%(p<0.001)$ for complex II (Fig. 1F). Morphine alone also increased states 2 (by $\sim 18 \%, p<0.001$ ) and 4 (by $\sim 16 \%, p<0.05$ ) respiration in liver mitochondria energized through complex II (Fig. 1B and F). In brain mitochondria energized with complex I substrates, cocaine and its combination with morphine decreased state 3 respiration by $\sim 15 \%(p<0.001)$ for cocaine and $\sim 31 \%(p<0.001)$ for Coc+Mor (Fig. 1C). Interestingly, the decrease evoked by Coc+Mor was significantly higher than that evoked by cocaine $(p<0.001)$ or morphine $(p<0.01)$ alone (Fig. 1C). Coc+Mor also increased state 4 respiration in brain mitochondria energized with complex I substrates, by $\sim 17 \%(p<0.01)$ (Fig. 1E).

Cocaine and Coc+Mor decreased RCR in brain and liver mitochondria energized with complex I and II substrates, although cocaine's effect on RCR in liver mitochondria energized with complex I substrates was not statistically significant (Fig. 1G and $\mathrm{H}$ ). In brain mitochondria, cocaine decreased RCR by $\sim 21 \%(p<0.001)$, for complex I, and by $\sim 19 \%(p<0.001)$ for complex II, whereas Coc+Mor decreased RCR by $\sim 33 \%(p<0.001)$ for complex I and $\sim 21 \%(p<0.001)$ for complex II (Fig. 1G). In liver mitochondria, cocaine decreased RCR by $\sim 21 \%$ (non-significant) for complex I, and by $\sim 38 \%(p<0.001)$ for complex II, whereas Coc+Mor decreased RCR by $\sim 29 \%(p<0.01)$ for complex I and $\sim 25 \%$ ( $p<0.01)$ for complex II (Fig. 1H). Morphine alone also decreased RCR in brain mitochondria energized through complex I (by $\sim 15 \% ; p<0.01$ ) and in liver mitochondria energized through complex II (by $\sim 33 \%$; $p<0.01$ ) (Fig. $1 \mathrm{G}$ and $\mathrm{H}$ ). The drugs or their combination did not significantly affect $\mathrm{ADP} / \mathrm{O}$ (Fig. $1 \mathrm{I}$ and $\mathrm{J}$ ), nor state 2 respiration in brain mitochondria (Fig. 1A), or state 3 respiration in liver mitochondria (Fig. 1D).

In liver mitochondria energized with complex I or complex II substrates, cocaine and $\mathrm{Coc}+$ Mor increased respiration upon addition of oligomycin (Fig. 2B). Cocaine increased respiration in the presence of oligomycin by $\sim 22 \%$ ( $p<0.001$ ), for complex I, and by $\sim 35 \%$ ( $p<0.001)$ for complex II, whereas for Coc+Mor the decrease was $\sim 30 \%(p<0.001)$ for complex I and $\sim 35 \%$ ( $p<0.001)$ for complex II (Fig. 2B). Morphine alone slightly decreased respiration upon oligomycin addition (by $\sim 15 \% ; p<0.01$ ) in liver mitochondria energized with complex I substrates (Fig. 2B). Cocaine and Coc+Mor induced a decrease in FCCP-stimulated (uncoupled) respiration in brain and liver mitochondria energized with complex I substrates (Fig. 2C and D). In brain mitochondria, the decrease in FCCPstimulated respiration induced by cocaine was $\sim 22 \%(p<0.001)$, whereas for Coc+Mor the decrease was $\sim 28 \%$ ( $p<0.001$ ) (Fig. 2C). In liver mitochondria, cocaine decreased FCCP-stimulated respiration by $\sim 25 \%(p<0.001)$, whereas for Coc+Mor the decrease was $\sim 30 \%$ ( $p<0.001$ ) (Fig. 2D).

The selective increase in states 2 and 4 (Fig. 1B and F) and in respiration after oligomycin exposure (Fig. 2B) in liver mitochondria suggest that these mitochondrial preparations are more prone to proton leak caused by the drugs or their combination compared to brain mitochondria.

Cocaine and Coc+Mor induced a similar decrease in maximum potential $(\sim 7 \%, p<0.001)$ in brain mitochondria, only after energization with complex I substrates (Fig. 3A). In liver mitochondria, cocaine and $\mathrm{Coc}+\mathrm{Mor}$ induced a similar decrease in maximum potential $(\sim 9 \%, p<0.001)$ in brain mitochondria energized both with complex I or complex II substrates (Fig. 3B).

\subsection{Cocaine-induced inhibition of complex I is more evident in brain mitochondria}

In order to understand the direct effects of the drugs and their combination in the respiratory chain complexes, independently of mitochondrial physiology, we incubated mitochondrial fractions with cocaine and/or morphine and measured oxygen consumption after energization with NADH (for complex I) and succinate in the presence of rotenone (for complex II). Cocaine and $\mathrm{Coc}+\mathrm{Mor}$ induced a decrease in respiratory activity, compared to control conditions, in brain (respectively, 60.4\% and 65.4\%) and liver (respectively, $46.7 \%$ and $47.9 \%$ ) mitochondrial fractions energized through complex I, but not in mitochondrial fractions energized through complex II (Fig. 4). Since mitochondrial respiration upon energization with succinate was not affected by the drugs or their combination, this result suggests that cocaine directly affected complex I activity, and did not affect complexes II, III or IV activities. Interestingly, brain and liver mitochondrial preparations differed in their respiratory activities upon energization with substrates for complex I and complex II. Brain mitochondria were found to be more dependent on complex I for respiration, since in these mitochondrial fractions oxygen consumption after incubation with NADH was $63.88 \pm 1.31 \mathrm{nmol} / \mathrm{min} / \mathrm{mg}$ protein, as compared to $40.21 \pm 4.11 \mathrm{nmol} / \mathrm{min} / \mathrm{mg}$ protein in liver mitochondria. When incubated with the complex II substrate, succinate, liver mitochondrial preparations consumed more oxygen than brain mitochondrial preparations, respectively at $36.03 \pm 3.92$ and $15.18 \pm 0.64 \mathrm{nmol} / \mathrm{min} / \mathrm{mg}$ protein. This represents an increase of 1.6 fold in complex I respiration in brain mitochondria, compared to liver mitochondria, and an increase of 2.4 fold in complex II respiration in liver mitochondria, compared to brain mitochondria. The ratio of complex I/complex II respiration was 4.2 in brain mitochondria, whereas in liver mitochondria respiration was similar upon complex I or II energization (Fig. 4). These results may explain the different impact of the drugs and their combination in brain and liver mitochondrial respirations, since complex I seems to be the most affected by cocaine and Coc+Mor, and brain mitochondria seem to be more dependent on complex I activity.

\section{Discussion}

Mitochondrial function and energy metabolism were previously found to be affected in brains of human cocaine abusers (Lehrmann et al., 2003), and cocaine was shown to influence mitochondrial function in different cell types (Devi and Chan, 1997; Yuan and 
Brain

A

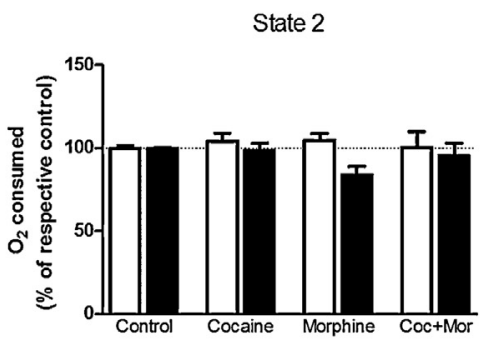

C

State 3

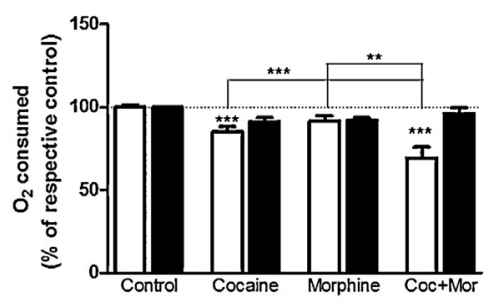

E

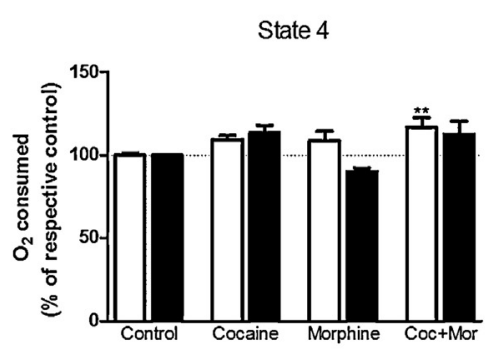

G

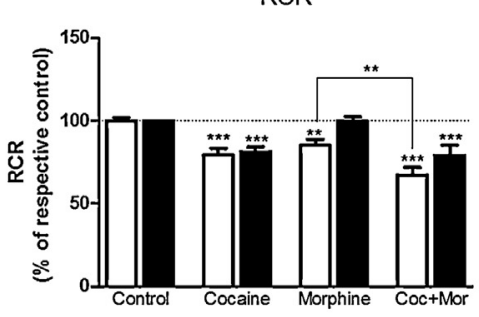

I

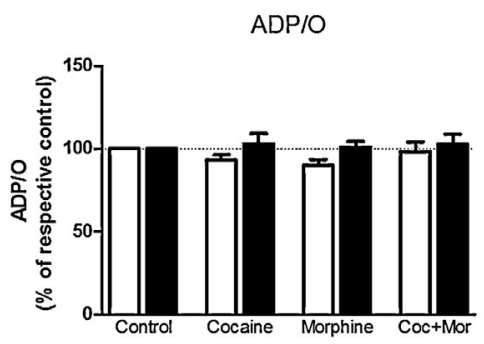

Liver

B

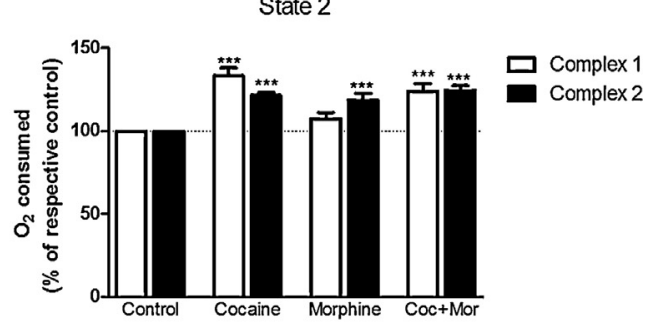

State 3

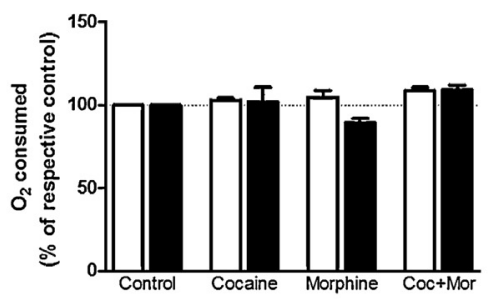

F

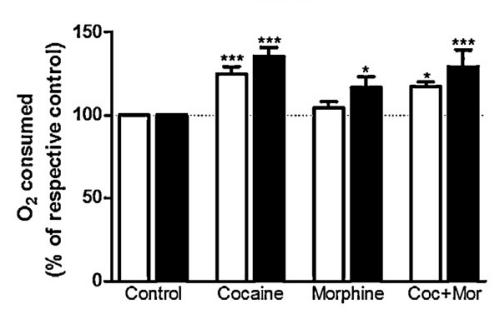

H

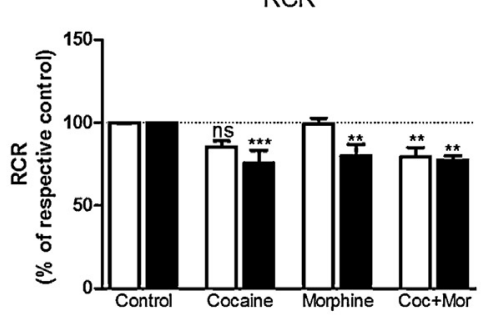

J

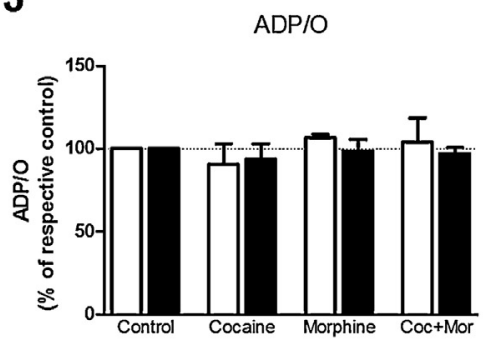

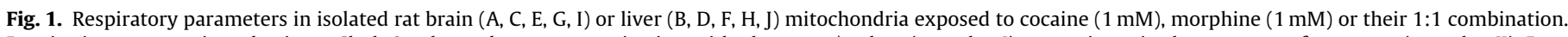

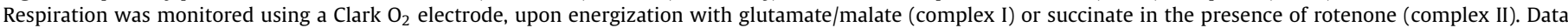

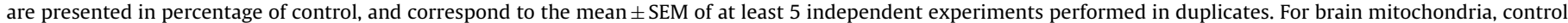

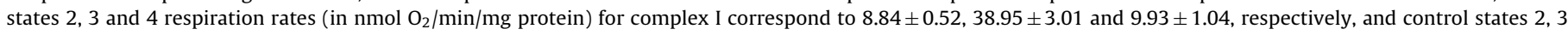

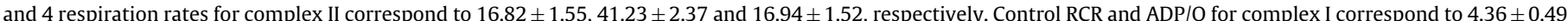

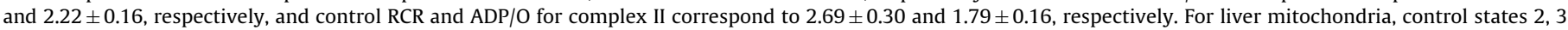

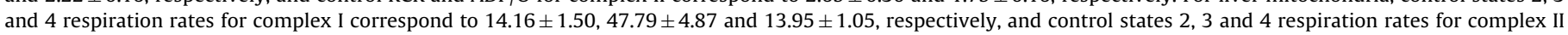

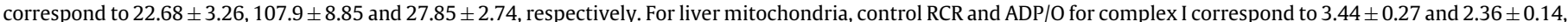

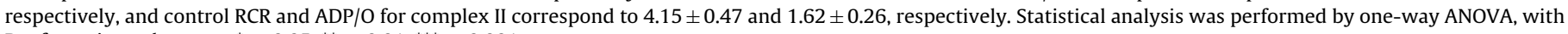
Bonferroni post hoc test: ${ }^{*} p<0.05,{ }^{* *} p<0.01,{ }^{* * *} p<0.001$. 

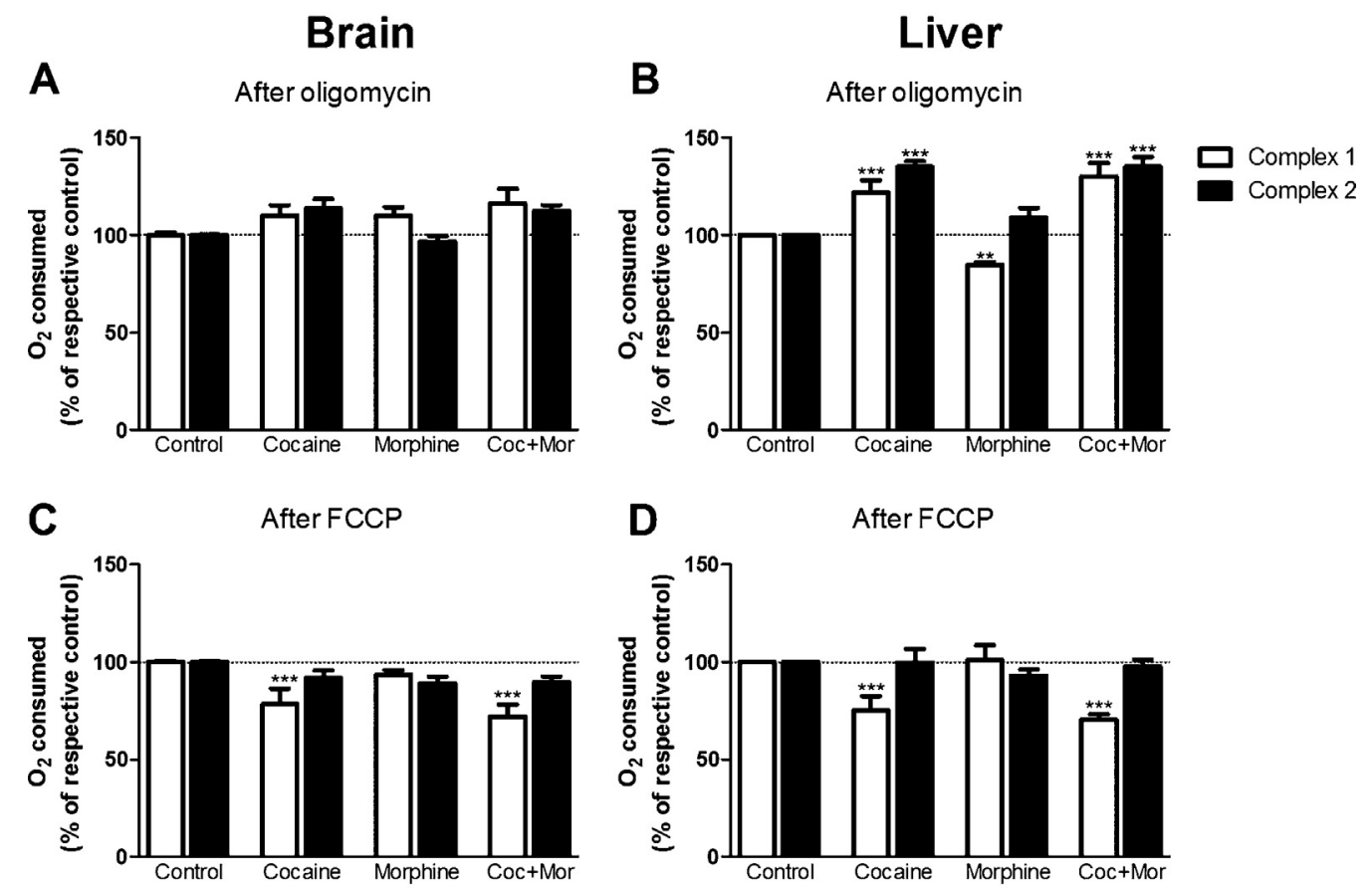

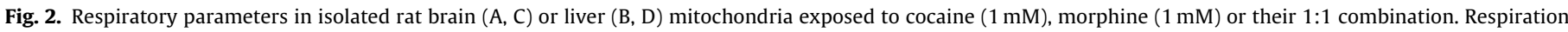

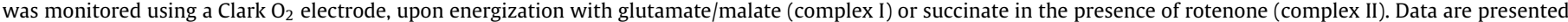

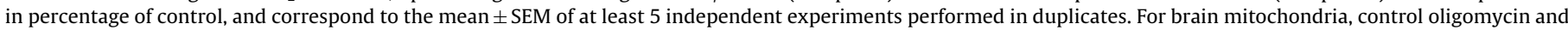

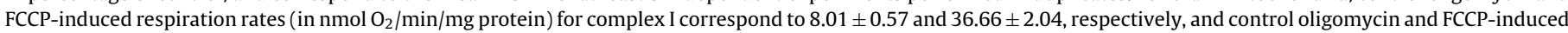

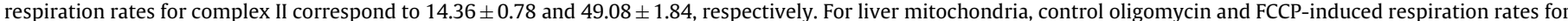

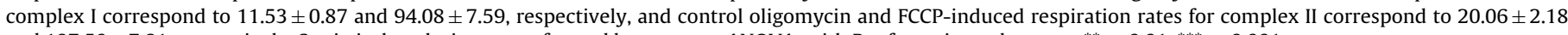
and $197.50 \pm 7.81$, respectively. Statistical analysis was performed by one-way ANOVA, with Bonferroni post hoc test: ${ }^{* *} p<0.01$, ${ }^{* * *} p<0.001$.

Acosta, 1996). Furthermore, cocaine is often co-used with opioids, such as heroin, in a combination commonly known as speedball. This combination also induces mitochondrial dysfunction, due to chemical or pharmacodynamic interactions between the drugs (Cunha-Oliveira et al., 2010).

Neurotoxicity of cocaine has been associated with mitochondrial-mediated apoptotic cell death, including changes in Bcl-2 and Bax expression (Dey et al., 2007; Lepsch et al., 2009; Xiao and Zhang, 2008), loss of mitochondrial potential, cytochrome c release and activation of initiator caspases from the intrinsic pathway (Cunha-Oliveira et al., 2008). Cocaine has been shown to induce hepatotoxicity (Van Thiel and Perper, 1992) and mitochondrial dysfunction in hepatic cells (Devi and Chan, 1997). However, much less is known about the direct effects of cocaine on the bioenergetics of brain mitochondria. The objective of the present study was to understand the specific effects of cocaine, morphine and $\mathrm{Coc}+\mathrm{Mor}$ in brain and liver mitochondria.

Concentrations of drugs used in this study were chosen after a dose-response preliminary study (data not shown). Cocaine plasma concentrations in human cocaine abusers have been found in the range between $0.3 \mu \mathrm{M}$ and $1 \mathrm{mM}$ (Heard et al., 2008; Yuan and Acosta, 2000). The concentration used in the present study ( $1 \mathrm{mM}$ ) or higher concentrations (up to $7 \mathrm{mM}$ ) have been previously used in in vitro studies by other investigators (Badisa et al., 2010; Garcia et al., 2012; Yuan and Acosta, 2000) (e.g. Boess et al., 2000). Morphine was used in the same concentration, to maintain
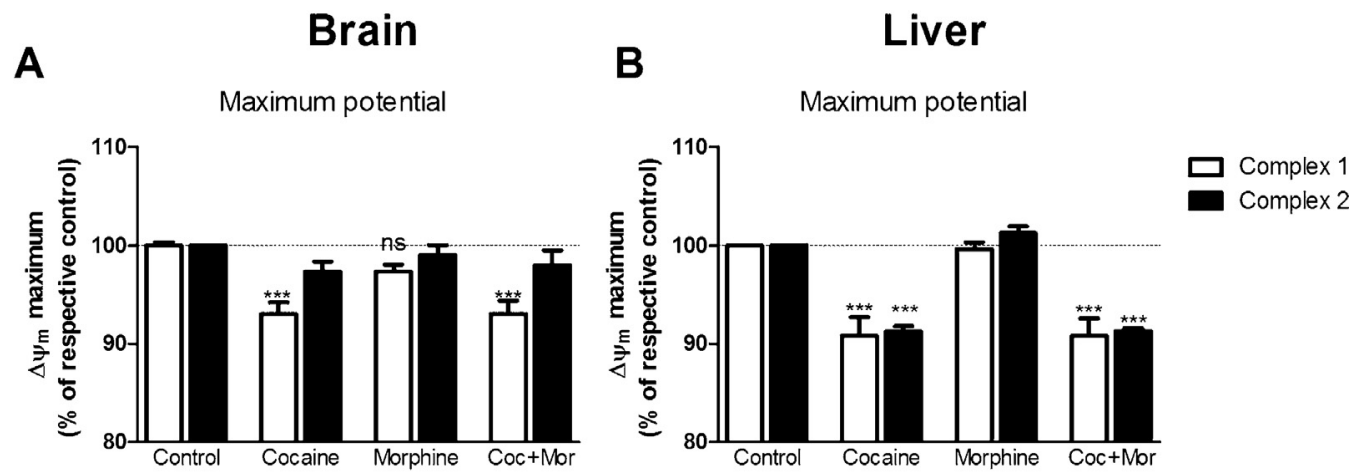

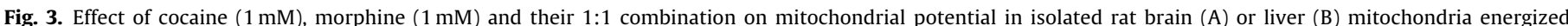

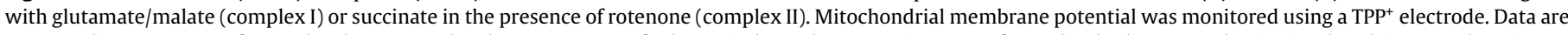

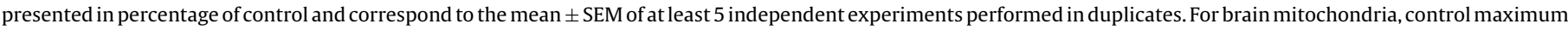

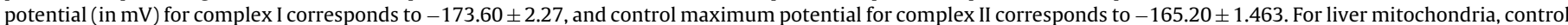

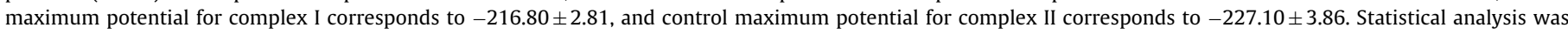
performed by one-way ANOVA, with Bonferroni post hoc test: ${ }^{* * *} p<0.001$ compared to control. 


\section{Brain}

A

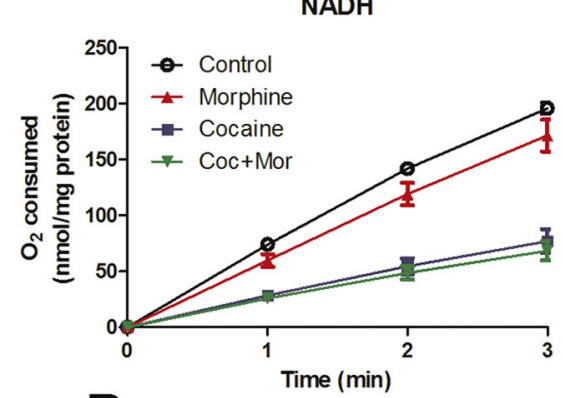

B

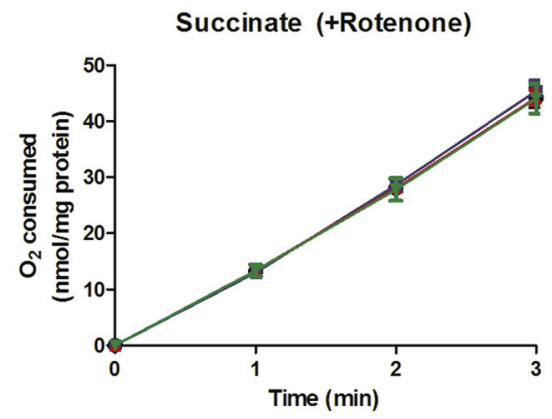

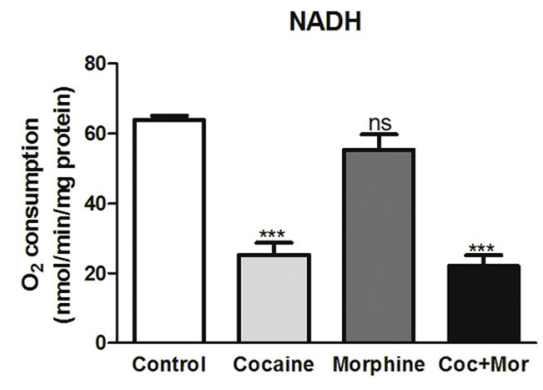

Succinate (+Rotenone)

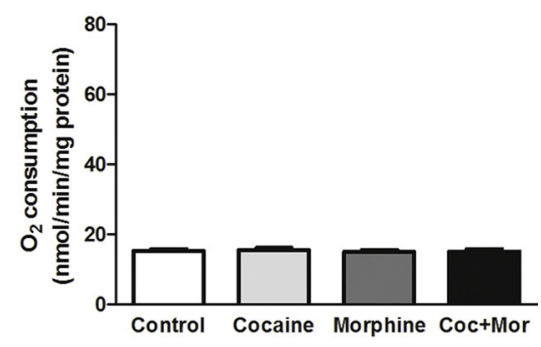

\section{Liver}

NADH

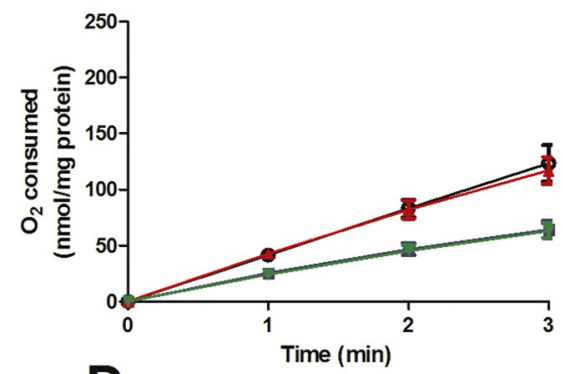

Succinate (+Rotenone)

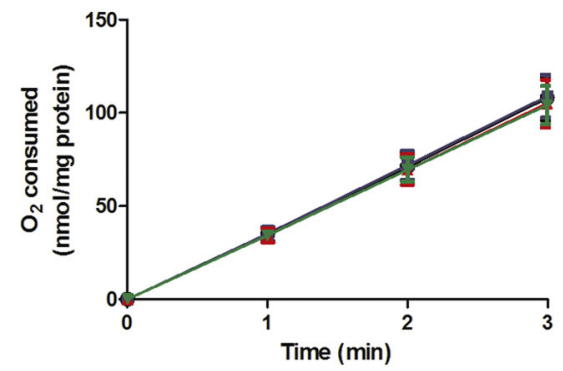

NADH

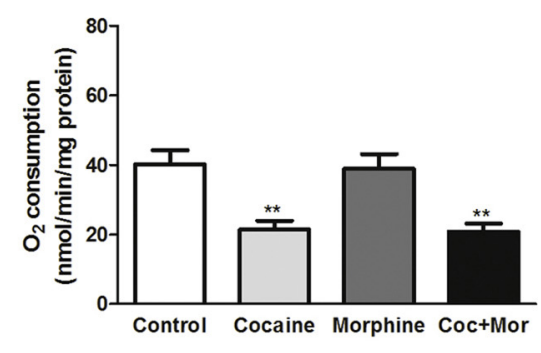

Succinate (+Rotenone)

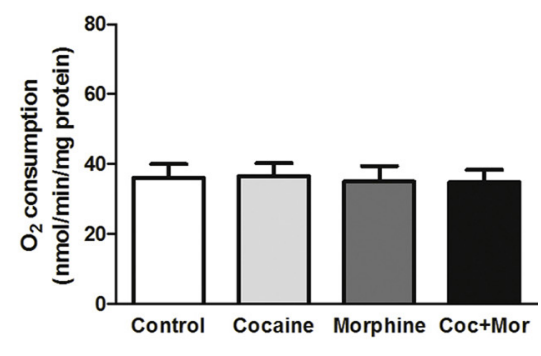

Fig. 4. Effect of cocaine $(1 \mathrm{mM})$, morphine $(1 \mathrm{mM})$ and their $1: 1$ combination on the respiratory activity in brain $(A, B)$ and liver (C, D) mitochondrial fractions. Respiration was monitored using a Clark $\mathrm{O}_{2}$ electrode, upon energization with NADH (complex I) or succinate in the presence of rotenone (complex II). The data presented correspond to the mean \pm SEM of at least 5 independent experiments performed in duplicates. Statistical analysis was performed by one-way ANOVA, with Bonferroni post hoc test: ${ }^{* *} p<0.01,{ }^{* * *} p<0.001$.

the $1: 1$ proportion between cocaine and morphine, at which cocaine:morphine adducts are formed (Garrido et al., 2007). At this concentration, morphine was shown to be toxic to neuronal-like PC12 cells (Oliveira et al., 2002) and in an even higher concentration $(4 \mathrm{mM})$ it induced the activation of the mitochondrial apoptotic pathway in SH-SY5Y cells (Lin et al., 2009). Plasma concentrations of morphine in animal models of addiction may achieve $2.5 \mathrm{mM}$ (Roy et al., 1998), although the peak plasma levels found in morphinerelated deaths were generally reported to range from $0.20-2.3 \mathrm{mg} / \mathrm{L}$ (Baselt, 2000), corresponding to 0.35-9.8 $\mu \mathrm{M}$. However, morphine 
and other drugs may accumulate in intracellular compartments (Bickel, 2005; Bouw et al., 2000; Hammarlund-Udenaes et al., 2008; Steinberg, 1994), and, therefore, may directly affect mitochondria. This also implicates that the reported blood levels of morphine are certainly lower than the intracellular concentrations. Although we used a high concentration of morphine, it seems to be well tolerated by brain and liver mitochondria, since only slight effects have been observed.

\subsection{Cocaine cytotoxicity and mitochondrial dysfunction in brain and liver}

Mitochondrial gene expression was found to be down-regulated in the brain, upon exposure to cocaine (Dietrich et al., 2004) and a functional respiratory chain seems to be required for cocaine's toxicity (Cunha-Oliveira et al., 2006). The involvement of complex I in cocaine's toxicity was suggested by the decrease in its activity and subunit expression observed after exposure to cocaine (Devi and Chan, 1997; Dietrich et al., 2004; Yuan and Acosta, 2000).

Our present results show that cocaine directly affects the respiratory chain in a different way in liver and brain mitochondria, but with a particular impact in complex I-mediated respiration in both tissues.

It is important to note that cocaine may interact directly with mitochondria and other intracellular targets (Heard et al., 2008), accumulating inside the cell due to its positive charge at physiological $\mathrm{pH}$. In the present work, cocaine decreased state 3 respiration in brain (but not in liver) mitochondria energized with complex I substrates and decreased FCCP-stimulated respiration and mitochondrial membrane potential in brain and liver mitochondria energized through complex I. The decrease of $\Delta \Psi$ induced by cocaine in liver mitochondria energized with succinate may not be explained by the effect of cocaine in respiration, but may be due to a direct dissipative charge effect, caused by the positive charge of cocaine at physiological pH (Sulzer and Rayport, 1990), which enables the accumulation of cocaine inside the mitochondria. Cocaine has also been shown to affect mitochondrial potential in rat cortical neurons (Cunha-Oliveira et al., 2006, 2010) and in cardiomyocytes (Xiao et al., 2000).

Our results also suggest that cocaine had a direct effect on complex I activity in brain and liver mitochondria, since this drug induced a decrease in oxygen consumption in mitochondrial fractions energized with NADH, and, thus, the effect of cocaine on complex I was not just due to an indirect effect, by acting on mitochondrial NADH production. The fact that succinate-mediated respiration in mitochondrial fractions was not affected by cocaine or Coc+Mor indicates that complexes II, III and IV were not affected in these conditions.

In liver mitochondria, cocaine affected respiration at states 2 and 4 , and after addition of oligomycin and FCCP. The increase observed in states 2 and 4 respiration in mitochondria exposed to cocaine suggests an augmentation of the passive transport of $\mathrm{H}^{+}$into the mitochondria, which is also supported by the increase in respiration observed in the presence of oligomycin, and may be possibly due to a bilateral damage in phospholipids (Videira et al., 2001).

Previous studies reported that liver mitochondrial bioenergetics was affected by in vivo cocaine administration, as observed by a decrease in state 3 respiration and RCR of hepatic mitochondria from rats (Devi and Chan, 1997) and mice (Leon-Velarde et al., 1992), and also by a decrease of the activity of complexes I, II/III, and IV (Devi and Chan, 1997). Importantly, cocaine metabolism may also contribute to the effects of in vivo cocaine administration, since the cocaine metabolites norcocaine and $\mathrm{N}-\mathrm{OH}$-norcocaine were shown to be more effective affecting state 3 , state 4 , RCR, and $\mathrm{ADP} / \mathrm{O}$ in mitochondria isolated from rat liver, compared to cocaine itself (Boess et al., 2000). The formation of cocaine-protein adducts that occurs primarily with proteins located in mitochondria and microsomal fractions, such as HSP-60 and transferrin, may also explain cocaine's mitochondrial effects (Ndikum-Moffor and Roberts, 2003).

\subsection{Morphine cytotoxicity and mitochondrial dysfunction in brain and liver}

Morphine is clinically used for the treatment of severe pain (Bekheet, 2010), and is also the active metabolite of heroin, mediating many of its effects (Cunha-Oliveira et al., 2008).

Heroin was previously shown to decrease mitochondrial potential and induce mitochondrial-associated apoptosis in rat cortical neurons (Cunha-Oliveira et al., 2007). Morphine ( $4 \mathrm{mM}$ ) was shown to promote apoptosis in SH-SY5Y cells, via activation of mitochondria-dependent pathway (Lin et al., 2009).

In the present study, only when succinate was used as a substrate in liver mitochondria, morphine increased states 2 and 4 respiration, and decreased $\mathrm{RCR}$ and respiration after exposure to oligomycin, suggesting an increase in the passive transport of $\mathrm{H}^{+}$into the mitochondria. In brain mitochondria, morphine only decreased RCR when glutamate/malate were used as substrates. Despite the high morphine concentration used in this study, it seemed to be well tolerated by brain and liver mitochondria.

\subsection{Speedball and mitochondrial dysfunction}

As previously mentioned, combination of heroin and cocaine (speedball) is very common (European Monitoring Center for Drugs and Drug Addiction, 2009) and the molecular effects of this combination are poorly understood. The effects of speedball may differ from the effects of each drug alone (Cunha-Oliveira et al., 2010), due to the formation of adducts between cocaine and morphine (Garrido et al., 2007) that may alter their chemical properties.

Very few publications describe the molecular effects of Coc+Mor combinations. In a previous study from our laboratory, we showed that cocaine-heroin combinations induced an increase in the number of dead cells, together with mitochondrial depolarization, ATP loss and cytochrome c release, in rat cortical neurons (CunhaOliveira et al., 2010). In that study, the drugs were administered to cultured cells in two different ways: sequentially, as separate stock solutions (heroin+cocaine), and simultaneously, using a solution containing both drugs (heroin:cocaine), prepared from a mixture of drugs in the solid forms. Both these preparations were expected to contain some proportion of morphine, due to spontaneous hydrolysis of the acetyl groups of heroin (Cunha-Oliveira et al., 2008). The intracellular pathways of neurotoxicity elicited by the two combinations were different, which may be attributed to the increased in chemical interaction between the molecules in the solution containing both drugs, used in the simultaneous administration (Cunha-Oliveira et al., 2010). In the present work, cocaine and morphine were incubated sequentially (1:1) from separate stock solutions to obtain the combination Coc+Mor, possibly underlying a low extent of cocaine:morphine adduct formation.

The drug combination Coc+Mor had a greater effect on state 3 respiration of brain mitochondria, compared to each drug per se. In most of the other parameters, the effects of Coc+Mor in brain mitochondria were similar to those of cocaine alone, including the decreases in RCR, FCCP-stimulated respiration, mitochondrial potential and complex I-stimulated respiratory activity.

The effects of Coc+Mor in the bioenergetics of liver mitochondria were also similar to the effects of cocaine alone, namely the increases in respiration in states 2, 4 and after oligomycin, and the decreases in RCR, FCCP-stimulated respiration, maximum potential and complex I-stimulated respiratory activity. 
Together, our results suggest that in brain and liver mitochondria the effects of Coc+Mor (1:1) combination in the respiratory chain and mitochondrial potential are similar to those induced by cocaine alone, as observed previously in cortical neurons (CunhaOliveira et al., 2010), although in brain mitochondria specific effects of the combination were also observed.

\subsection{Brain versus liver mitochondria}

When comparing the effects of cocaine and/or morphine in brain an liver mitochondria, we found that they were markedly different. Whereas in brain mitochondria energized through complex I cocaine and Coc+Mor mainly decreased state 3 respiration and RCR, in liver mitochondria states 2 and 4 were increased, and RCR was decreased in the presence of cocaine and Coc+Mor, regardless of the energetic substrate used. Furthermore, morphine did not present major effects in brain mitochondria, whereas in liver mitochondria, morphine increased states 2 and 4 respiration, and decreased RCR, only when the energetic substrate was succinate. Respiration after addition of FCCP was similarly decreased in brain and liver mitochondria upon exposure to cocaine or Coc+Mor, only for complex I substrates. In brain mitochondria, the decrease in maximum potential observed upon exposure to cocaine or Coc+Mor was only observed when the substrates were glutamate/malate. However, in liver mitochondria the decrease in mitochondrial potential observed upon cocaine or Coc+Mor exposure was similar for complex I and complex II substrates. Different effects observed in brain and liver mitochondria are possibly related with the different dependence on complex I for respiration in the two tissues, since brain mitochondria exhibited a 4.2 fold higher ratio of complex I/complex II respiration, whereas liver mitochondria respiration was almost equal upon complex I or II energization. Our results also suggest that liver mitochondria are more prone to proton leak caused by the drugs or their combination.

It is known that different tissues have different mitochondrial respiratory chain content, composition, activity and respiration velocity at state 3 (Benard et al., 2006). Brain mitochondria have the highest ATP production rate with complex I substrates, compared to heart and liver mitochondria, possibly related with their limited defenses against reactive oxygen species (ROS). This could contribute to a preference for a higher rate over a better yield, as a protective mechanism against ROS formation (Cocco et al., 2009), and may explain the differences observed in the present study.

\section{Conclusions}

In this work, we showed that brain and liver mitochondria respirations are differentially affected by cocaine, morphine and their combination, although in both tissues complex I inhibition seems to mediate many of cocaine's effects on respiration and mitochondrial potential. Liver mitochondria seem to be more prone to proton leak caused by the drugs or their combination. Morphine seems to be well tolerated by brain and liver mitochondria and the combination Coc+Mor presented similar effects to cocaine, except for a greater effect on state 3 respiration. Differences observed in brain and liver mitochondria may be related with the different dependence on complex I for respiration observed in each tissue. In summary, cocaine and Coc+Mor induced mitochondrial complex I dysfunction in isolated brain and liver mitochondria, with specific effects in each tissue.

\section{Conflict of interest statement}

The authors declare that there are no conflicts of interest.

\section{Acknowledgements}

The authors are grateful to Ana Carolina Moreira and Renato $\mathrm{X}$. Santos from CNC, University of Coimbra, for providing isolated brain mitochondria for some of the experiments. This work was supported by Grant III/34/2008 from Instituto de Investigação Interdisciplinar da Universidade de Coimbra and Grants PTDC/AGR-ALI/108326/2008 and PEst-C/SAU/LA0001/2011 funded by Fundação para a Ciência e a Tecnologia (FCT), Portugal, and cofinanced by: "COMPETE - Programa Operacional Factores de Competitividade”, QREN and European Union (FEDER - Fundo Europeu de Desenvolvimento Regional). T.C.-O. was supported by the FCT postdoctoral fellowship SFRH/BPD/34711/2007 and AMS by the PhD fellowship from FCT, ref SFRH/BD/76086/2011, cofinanced by POPH - Programa Operacional Potencial Humano, QREN and European Union.

\section{References}

Badisa, R.B., Darling-Reed, S.F., Goodman, C.B., 2010. Cocaine induces alterations in mitochondrial membrane potential and dual cell cycle arrest in rat c6 astroglioma cells. Neurochemical Research 35, 288-297.

Bandettini Di Poggio, A., Fornai, F., Paparelli, A., Pacini, M., Perugi, G., Maremmani, I., 2006. Comparison between heroin and heroin-cocaine polyabusers: a psychopathological study. Annals of the New York Academy of Sciences 1074, 438-445.

Baselt, R.C., 2000. Disposition of Toxic Drugs \& Chemicals in Man, 5th ed. Chemical Toxicology Institute, California.

Bekheet, S.H., 2010. Morphine sulphate induced histopathological and histochemical changes in the rat liver. Tissue and Cell 42, 266-272.

Benard, G., Faustin, B., Passerieux, E., Galinier, A., Rocher, C., Bellance, N., Delage, J.P., Casteilla, L., Letellier, T., Rossignol, R., 2006. Physiological diversity of mitochondrial oxidative phosphorylation. American Journal of Physiology: Cell Physiology 291, C1172-C1182.

Bickel, U., 2005. How to measure drug transport across the blood-brain barrier. NeuroRx 2, 15-26.

Boess, F., Ndikum-Moffor, F.M., Boelsterli, U.A., Roberts, S.M., 2000. Effects of cocaine and its oxidative metabolites on mitochondrial respiration and generation of reactive oxygen species. Biochemical Pharmacology 60, 615-623.

Bouw, M.R., Gardmark, M., Hammarlund-Udenaes, M., 2000. Pharmacokineticpharmacodynamic modelling of morphine transport across the blood-brain barrier as a cause of the antinociceptive effect delay in rats-a microdialysis study. Pharmaceutical Research 17, 1220-1227.

Chance, B., Williams, G.R., 1956. The respiratory chain and oxidative phosphorylation. Advances in enzymology and related subjects of biochemistry 17,65-134

Cocco, T., Pacelli, C., Sgobbo, P., Villani, G., 2009. Control of OXPHOS efficiency by complex I in brain mitochondria. Neurobiology of Aging 30, 622-629.

Cunha-Oliveira, T., Rego, A.C., Cardoso, S.M., Borges, F., Swerdlow, R.H., Macedo, T., de Oliveira, C.R., 2006. Mitochondrial dysfunction and caspase activation in rat cortical neurons treated with cocaine or amphetamine. Brain Research 1089, 44-54.

Cunha-Oliveira, T., Rego, A.C., Garrido, J., Borges, F., Macedo, T., Oliveira, C.R., 2007. Street heroin induces mitochondrial dysfunction and apoptosis in rat cortical neurons. Journal of Neurochemistry 101, 543-554.

Cunha-Oliveira, T., Rego, A.C., Garrido, J., Borges, F., Macedo, T., Oliveira, C.R., 2010. Neurotoxicity of heroin-cocaine combinations in rat cortical neurons. Toxicology 276, 11-17

Cunha-Oliveira, T., Rego, A.C., Oliveira, C.R., 2008. Cellular and molecular mechanisms involved in the neurotoxicity of opioid and psychostimulant drugs. Brain Research Reviews 58, 192-208.

Devi, B.G., Chan, A.W., 1997. Impairment of mitochondrial respiration and electron transport chain enzymes during cocaine-induced hepatic injury. Life Sciences $60,849-855$.

Dey, S., Mactutus, C.F., Booze, R.M., Snow, D.M., 2007. Cocaine exposure in vitro induces apoptosis in fetal locus coeruleus neurons by altering the Bax/Bcl-2 ratio and through caspase-3 apoptotic signaling. Neuroscience 144, 509-521.

Dietrich, J.B., Poirier, R., Aunis, D., Zwiller, J., 2004. Cocaine downregulates the expression of the mitochondrial genome in rat brain. Annals of the New York Academy of Sciences 1025, 345-350.

Estabrook, R., 1967. Membranes of Mitochondria and Chloroplasts. Van Nostrand, New York172-212.

European Monitoring Center for Drugs and Drug Addiction, 2008. Annual Report 2008. The state of the drugs problem in Europe.

European Monitoring Center for Drugs and Drug Addiction, 2009. Polydrug use: patterns and responses.

Garcia, R.C., Dati, L.M., Fukuda, S., Torres, L.H., Moura, S., de Carvalho, N.D., Carrettiero, D.C., Camarini, R., Levada-Pires, A.C., Yonamine, M., Negrini-Neto, O., Abdalla, F.M., Sandoval, M.R., Afeche, S.C., Marcourakis, T., 2012. Neurotoxicity of anhydroecgonine methyl ester, a crack cocaine pyrolysis product. Toxicological Sciences $128,223-234$. 
Garrido, J.M., Marques, M.P., Silva, A.M., Macedo, T.R., Oliveira-Brett, A.M., Borges, F. 2007. Spectroscopic and electrochemical studies of cocaine-opioid interactions. Analytical and Bioanalytical Chemistry.

Gornall, A.G., Bardawill, C.J., David, M.M., 1949. Determination of serum proteins by means of the Biuret reaction. Journal of Biological Chemistry 177, 751-766.

Hammarlund-Udenaes, M., Friden, M., Syvanen, S., Gupta, A., 2008. On the rate and extent of drug delivery to the brain. Pharmaceutical Research 25, 17371750.

Heard, K., Palmer, R., Zahniser, N.R., 2008. Mechanisms of acute cocaine toxicity. Open Pharmacology Journal 2, 70-78.

Kamo, N., Muratsugu, M., Hongoh, R., Kobatake, Y., 1979. Membrane potential of mitochondria measured with an electrode sensitive to tetraphenyl phosphonium and relationship between proton electrochemical potential and phosphorylation potential in steady state. Journal of Membrane Biology 49, 105-121.

Lehrmann, E., Oyler, J., Vawter, M.P., Hyde, T.M., Kolachana, B., Kleinman, J.E., Huestis, M.A., Becker, K.G., Freed, W.J., 2003. Transcriptional profiling in the human prefrontal cortex: evidence for two activational states associated with cocaine abuse. Pharmacogenomics Journal 3, 27-40.

Leon-Velarde, F., Huicho, L., Monge, C., 1992. Effects of cocaine on oxygen consumption and mitochondrial respiration in normoxic and hypoxic mice. Life Sciences $50,213-218$.

Lepsch, L.B., Munhoz, C.D., Kawamoto, E.M., Yshii, L.M., Lima, L.S., Curi-Boaventura, M.F., Salgado, T.M., Curi, R., Planeta, C.S., Scavone, C., 2009. Cocaine induces cell death and activates the transcription nuclear factor kappa-b in pc12 cells. Molecular Brain 2, 3.

Leri, F., Bruneau, J., Stewart, J., 2003. Understanding polydrug use: review of heroin and cocaine co-use. Addiction 98, 7-22.

Lin, X., Wang, Y.J., Li, Q., Hou, Y.Y., Hong, M.H., Cao, Y.L., Chi, Z.Q., Liu, J.G., 2009. Chronic high-dose morphine treatment promotes SH-SY5Y cell apoptosis via c-Jun N-terminal kinase-mediated activation of mitochondria-dependent pathway. FEBS Journal 276, 2022-2036.

Moreira, A.C., Silva, A.M., Santos, M.S., Sardao, V.A., 2012. Resveratrol affects differently rat liver and brain mitochondrial bioenergetics and oxidative stress in vitro: investigation of the role of gender. Food and Chemical Toxicology.

Moreno, A.J., Oliveira, P.J., Nova, C.D., Alvaro, A.R., Moreira, R.A., Santos, S.M., Macedo, T., 2007. Unaltered hepatic oxidative phosphorylation and mitochondrial permeability transition in wistar rats treated with nimesulide: relevance for nimesulide toxicity characterization. Journal of Biochemical and Molecular Toxicology 21, 53-61.

Ndikum-Moffor, F.M., Roberts, S.M., 2003. Cocaine-protein targets in mouse liver. Biochemical Pharmacology 66, 105-113.

Oliveira, M.T., Rego, A.C., Morgadinho, M.T., Macedo, T.R., Oliveira, C.R., 2002. Toxic effects of opioid and stimulant drugs on undifferentiated PC12 cells. Annals of the New York Academy of Sciences 965, 487-496.

Ranaldi, R., Munn, E., 1998. Polydrug self-administration in rats: cocaine-heroin is more rewarding than cocaine-alone. Neuroreport 9, 2463-2466.

Rosenthal, R.E., Hamud, F., Fiskum, G., Varghese, P.J., Sharpe, S., 1987. Cerebra ischemia and reperfusion: prevention of brain mitochondrial injury by lidoflazine. Journal of Cerebral Blood Flow and Metabolism 7, 752-758.

Roy, S., Cain, K.J., Chapin, R.B., Charboneau, R.G., Barke, R.A., 1998. Morphine modulates NF kappa B activation in macrophages. Biochemical and Biophysical Research Communications 245, 392-396.

Steinberg, T.H., 1994. Cellular transport of drugs. Clinical Infectious Diseases 19 916-921.

Sulzer, D., Rayport, S., 1990. Amphetamine and other psychostimulants reduce $\mathrm{pH}$ gradients in midbrain dopaminergic neurons and chromaffin granules: a mechanism of action. Neuron 5, 797-808.

Van Thiel, D.H., Perper, J.A., 1992. Hepatotoxicity associated with cocaine abuse. Recent Developments in Alcoholism 10, 335-341.

Videira, R.A., Antunes-Madeira, M.C., Madeira, V.M., 2001. Ethylazinphos interaction with membrane lipid organization induces increase of proton permeability and impairment of mitochondrial bioenergetic functions. Toxicology and Applied Pharmacology 175, 209-216.

Xiao, D., Zhang, L., 2008. Upregulation of Bax and Bcl-2 following prenatal cocaine exposure induces apoptosis in fetal rat brain. International Journal of Medical Sciences 5, 295-302.

Xiao, Y., He, J., Gilbert, R.D., Zhang, L., 2000. Cocaine induces apoptosis in feta myocardial cells through a mitochondria-dependent pathway. Journal of Pharmacology and Experimental Therapeutics 292, 8-14.

Yuan, C., Acosta Jr., D., 1996. Cocaine-induced mitochondrial dysfunction in primary cultures of rat cardiomyocytes. Toxicology 112, 1-10.

Yuan, C., Acosta Jr., D., 2000. Effect of cocaine on mitochondrial electron transport chain evaluated in primary cultures of neonatal rat myocardial cells and in isolated mitochondrial preparations. Drug and Chemical Toxicology 23 339-348. 